\title{
Conversion of $\alpha$-Halo-Aldehydes into Acylating Agents by an Internal Redox Reaction Catalyzed by Nucleophilic Carbenes.
}

Nathan T. Reynolds, Javier Read de Alaniz and Tomislav Rovis*

Department of Chemistry, Colorado State University

Fort Collins, Colorado 80523

\section{Supporting Information}

General Methods. All reactions were carried out under an atmosphere of argon in flame-dried glassware with magnetic stirring. Toluene was degassed with argon and passed through one column of neutral alumina and one column of Q5 reactant. Column chromatography was performed on EM Science silica gel 60 (230-400 mesh). Thin layer chromatography was performed on EM Science $0.25 \mathrm{~mm}$ silica gel 60-F plates. Visualization was accomplished with UV light, $\mathrm{KMnO}_{4}$, or aqueous ceric ammonium molybdate dips followed by heating.

Cyclohexanol was used as purchased from Mallinckrodt. All other alcohols and aniline were purchased from Aldrich Chemical Co. and used without further purification. $\alpha-$ Haloaldehydes were prepared by an adaptation of a literature procedure. ${ }^{1}$ Previously synthesized compounds matched reported spectra and new esters are characterized below.

Infrared spectra were obtained on a Nicolet Avatar 320 FT-IR spectrometer. ${ }^{1} \mathrm{H}$ NMR and spectra were recorded on a Varian 300, 400, or $500 \mathrm{MHz}$ spectrometer at ambient temperature. Data are reported as follows: chemical shift in parts per million $(\delta$, $\mathrm{ppm}$ ) from an internal standard [tetramethylsilane (TMS) or deuterated chloroform $\left(\mathrm{CDCl}_{3}\right)$ ], multiplicity $(\mathrm{s}=$ singlet, $\mathrm{d}=$ doublet, $\mathrm{t}=$ triplet, $\mathrm{q}=$ quartet, and $\mathrm{m}=$ multiplet $)$, integration, and coupling constant $(\mathrm{Hz}) .{ }^{13} \mathrm{C} N \mathrm{NMR}$ were recorded on a Varian spectrometer at 75,100 , or $125 \mathrm{MHz}$ at ambient temperature. Chemical shifts are reported in ppm from $\mathrm{CDCl}_{3}$ taken as $77.0 \mathrm{ppm}$. Mass spectra were obtained on Fisons VG Autospec.

General procedure for the internal redox reaction of $\boldsymbol{\alpha}$-halo-aldehydes: A flamedried round-bottomed flask was charged with azolium salt $(0.2 \mathrm{eq})$. Toluene (sufficient to attain a final concentration of $0.15 \mathrm{M}$ in substrate), triethylamine (1.0 eq), and alcohol (1.0 eq) were added via syringe and the solution was stirred at ambient temperature for 10 minutes. $\alpha$-Halo-aldehyde was then added via syringe and the reaction was stirred at room temperature for the time indicated. The reaction mixture was then subjected directly to column chromatography eluting with $99: 1$ hexane/ $\mathrm{Et}_{2} \mathrm{O}$ affording the pure product.

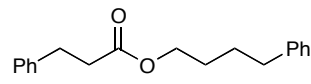

3-Phenyl-propionic acid 4-phenyl-butyl ester: $\mathrm{Rf}=0.40(9: 1$ $\mathrm{Hex} / \mathrm{EtOAc}) ;{ }^{1} \mathrm{H} \mathrm{NMR}\left(300 \mathrm{MHz}, \mathrm{CDCl}_{3}\right) \delta$ 7.20-7.36 (m, 10H),

\footnotetext{
${ }^{1}$ Halland, N.; Braunton, A.; Bachmann, S.; Marigo, M.; Jørgensen, K. A. J. Am. Chem. Soc. 2004, 126, 4790-4791.
} 
4.12-4.16 (m, 2H), $3.00(\mathrm{t}, 2 \mathrm{H}, J=7.7,8.1 \mathrm{~Hz}), 2.67(\mathrm{t}, 4 \mathrm{H}, J=8.1,7.3 \mathrm{~Hz}), 1.67-1.72$ $(\mathrm{m}, 4 \mathrm{H}) ;{ }^{13} \mathrm{C}$ NMR $\left(300 \mathrm{MHz}, \mathrm{CDCl}_{3}\right) \delta 172.7,141.9,140.4,128.4,128.3,128.2,128.1$, 126.1, 125.7, 65.8, 64.3, 36.0, 35.5, 31.0, 28.3, 27.7; IR (NaCl, neat), 1733, 1496, 1454, $1162 \mathrm{~cm}^{-1}$; HRMS (FAB+) calcd for $\mathrm{C}_{19} \mathrm{H}_{22} \mathrm{O}_{2}, 283.1698$, Found 283.1699.

3) $\int$ 3henyl-propionic acid 1-ethyl-prop-2-ynyl ester: $\mathrm{Rf}=0.45(9: 1$ Hex/EtOAc); ${ }^{1} \mathrm{H}$ NMR $\left(400 \mathrm{MHz}, \mathrm{CDCl}_{3}\right) \delta 7.24-7.33(\mathrm{~m}, 5 \mathrm{H}), 5.36(\mathrm{td}$, $1 \mathrm{H}, J=6.4,6.5,2.2,2.2,2.0 \mathrm{~Hz}), 3.01(\mathrm{t}, 2 \mathrm{H}, J=7.7,7.9 \mathrm{~Hz}), 2.68-2.74(\mathrm{~m}, 2 \mathrm{H}), 2.48$ $(\mathrm{d}, 1 \mathrm{H}, J=2.2 \mathrm{~Hz}), 1.76-1.86(\mathrm{~m}, 2 \mathrm{H}), 1.02(\mathrm{t}, 3 \mathrm{H}, J=7.3,7.5 \mathrm{~Hz}) ;{ }^{13} \mathrm{C}$ NMR $(100 \mathrm{MHz}$, $\left.\mathrm{CDCl}_{3}\right) \delta 171.8,140.3,128.5,128.3,126.3,81.0,73.5,64.9,35.8,30.8,27.8,9.2$; IR ( $\mathrm{NaCl}$, neat), 1739, 1455, $1159 \mathrm{~cm}^{-1}$; HRMS (FAB+) calcd for $\mathrm{C}_{14} \mathrm{H}_{16} \mathrm{O}_{2}, 217.1229$. Found 217.1230.

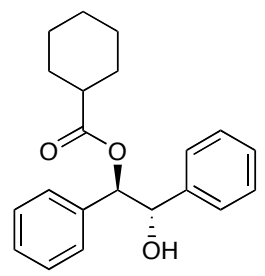

Cyclohexanecarboxylic acid 2-hydroxy 1,2-diphenyl-ethyl ester. $\mathrm{R}_{\mathrm{f}}$ $(4: 1$ hexane to ethyl acetate $)=0.5 ;[\alpha]_{\mathrm{D}}{ }^{23}=+5.8^{\circ}\left(\mathrm{CDCl}_{3}\right) ;$ HPLC analysis - Chiracel AD column 90:10 hexanes to isopropanol 0.5 $\mathrm{mL} / \mathrm{min}$. Major: 23.3 minutes, Minor: 29.0 minutes; ${ }^{1} \mathrm{H}$ NMR $(400 \mathrm{MHz}$, $\left.\mathrm{CDCl}_{3}\right) \delta 7.26(10 \mathrm{H}, \mathrm{m}), 5.87(1 \mathrm{H}, \mathrm{d}, J=6.4 \mathrm{~Hz}), 4.93(1 \mathrm{H}, \mathrm{d}, J=6.4$ $\mathrm{Hz}), 2.24-2.10(2 \mathrm{H}, \mathrm{m}) 1.78-1.56(5 \mathrm{H}, \mathrm{m}) 1.30-1.11(5 \mathrm{H}, \mathrm{m}) ;{ }^{13} \mathrm{C} \mathrm{NMR}$ $\left(400 \mathrm{MHz}, \mathrm{CDCl}_{3}\right) \delta 174.9,139.8,137.1,128.5,128.5,128.3,127.8,127.3,78.5,76.8$, 43.4, 28.9, 28.9, 25.9, 25.5; IR ( $\left.\mathrm{NaCl}, \mathrm{CH}_{2} \mathrm{Cl}_{2}\right)$ 3480, 2929, 2854, 1727, 1445; HRMS $(\mathrm{FAB}+)$ calcd for $\mathrm{C}_{21} \mathrm{H}_{24} \mathrm{O}_{3} 324.1725$. Found 324.1759. 
Spectra:

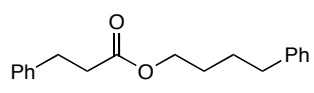

$\mathrm{nr}-4 \mathrm{j}$ une $-4 \mathrm{aPROD}$

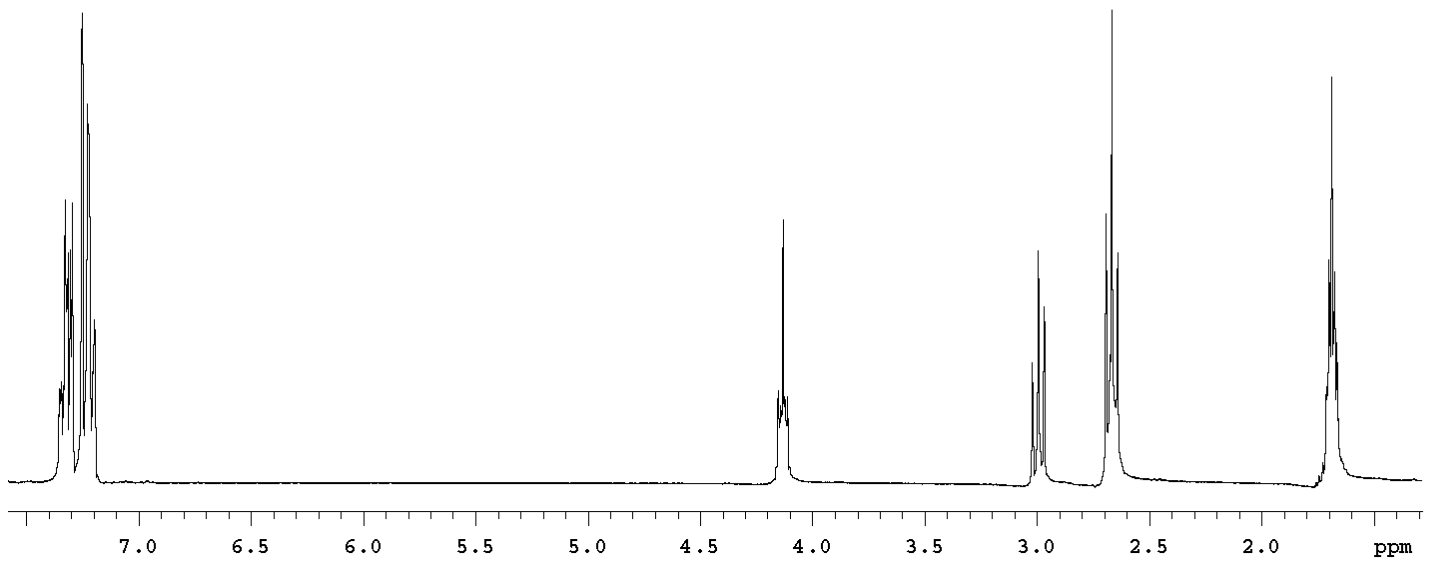

13C OBSERVE

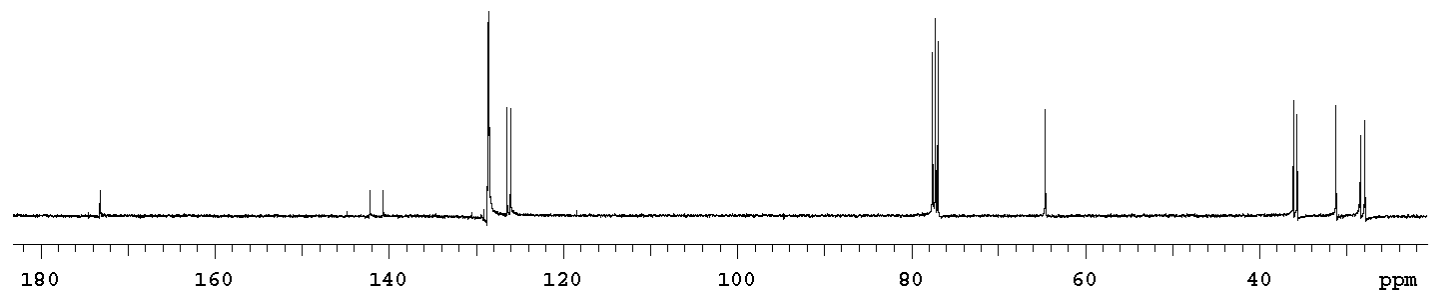


nr- 4 june-4b-PRODH1
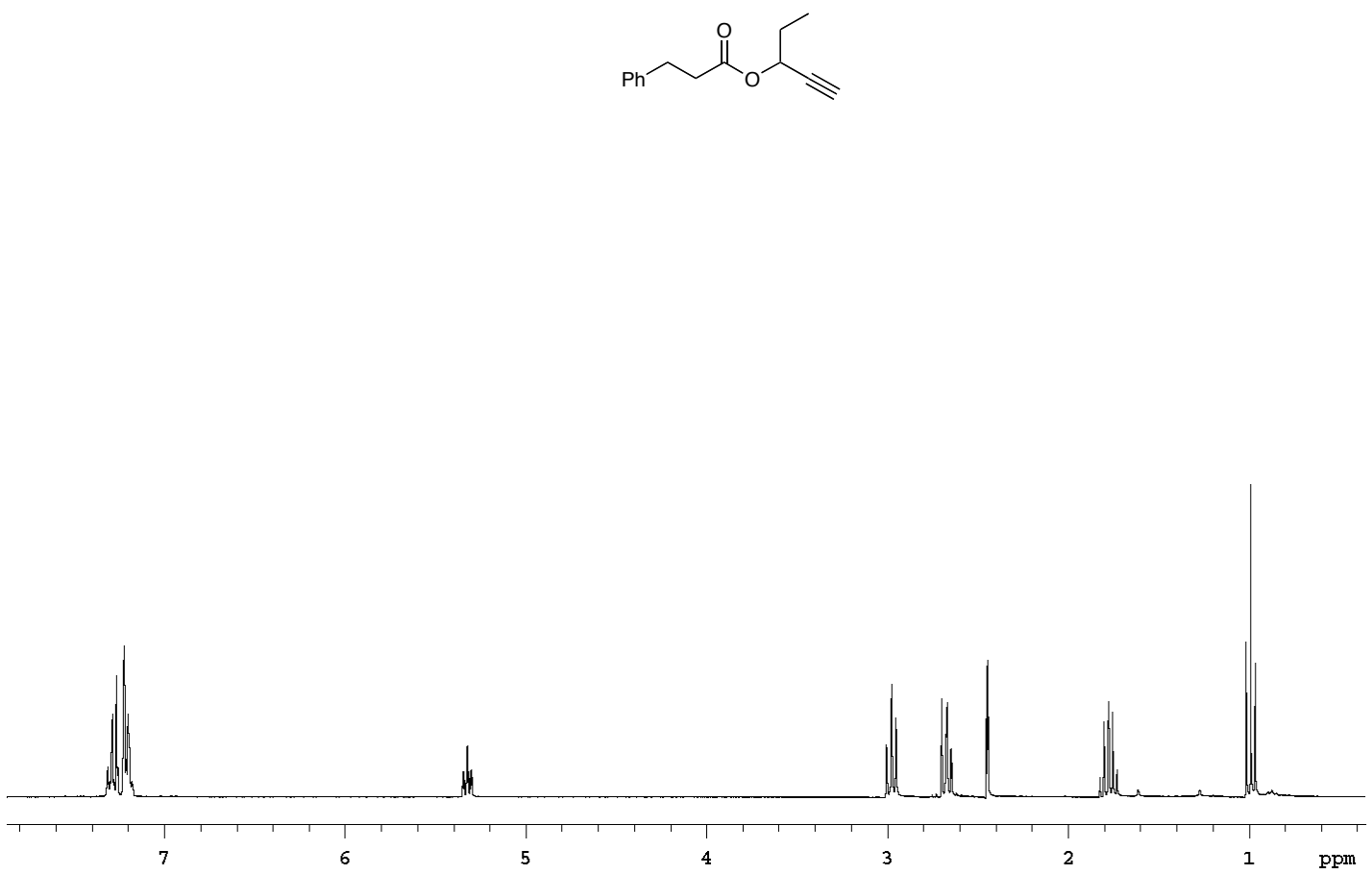

13C OBSERVE

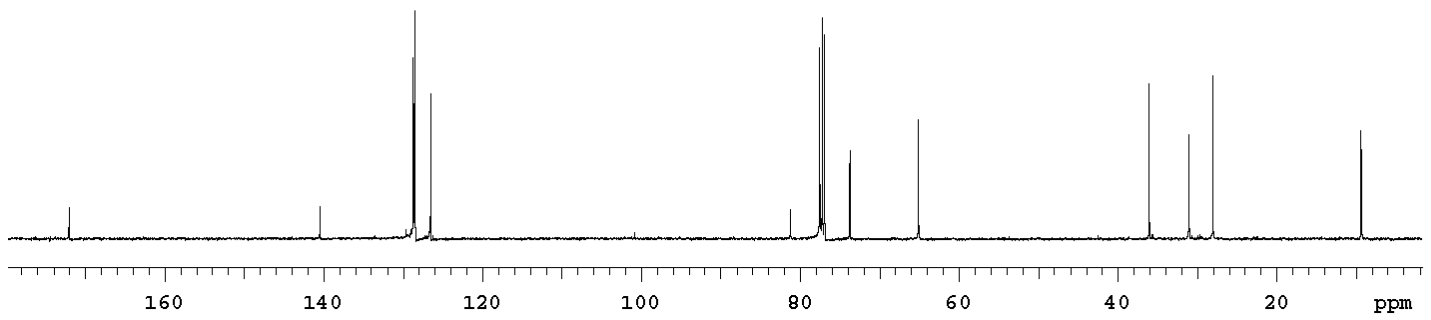



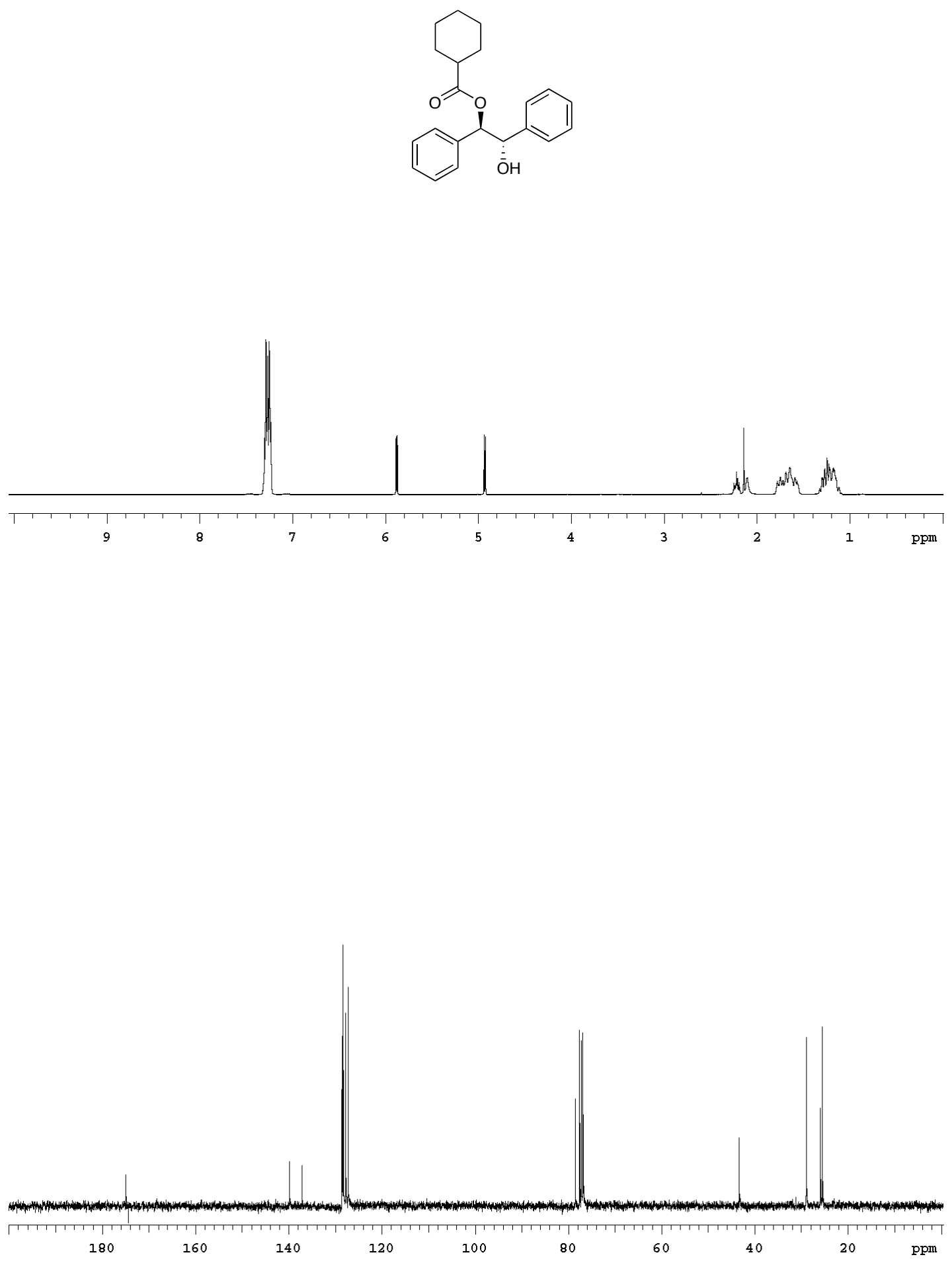